\title{
Can Massive Dark Haloes Destroy the Discs of Dwarf Galaxies?
}

\author{
B. Fuchs and O. Esquivel \\ Astronomisches Rechen-Institut am Zentrum für Astronomie der Universität Heidelberg, \\ Mönchhofstr. 12 - 14, 69120 Heidelberg, Germany \\ email: fuchs@ari.uni-heidelberg.de
}

\begin{abstract}
Recent high-resolution simulations together with theoretical studies of the dynamical evolution of galactic disks have shown that contrary to wide-held beliefs a 'live', dynamically responsive, dark halo surrounding a disk does not stabilize the disk against dynamical instabilities. We generalize Toomre's Q stability parameter for a disk-halo system and show that if a disk, which would be otherwise stable, is embedded in a halo, which is too massive and cold, the combined disk-halo system can become locally Jeans unstable. The good news is, on the other hand, that this will not happen in real dark haloes, which are in radial hydrostatic equilibrium. Even very low-mass disks are not prone to such dynamical instabilities.
\end{abstract}

Keywords. galaxies: kinematics and dynamics, galaxies: halos

\section{Introduction}

The classical paradigm is that at given total mass of the system a galactic disk is stabilized against local dynamical Jeans instabilities, if it is embedded in a dark halo. This can be seen for instance from Toomre's $Q_{\mathrm{T}}$ stability index for a stellar disk,

$$
Q_{\mathrm{T}}=\frac{\kappa \sigma_{\mathrm{d}}}{3.36 G \Sigma_{\mathrm{d}}}
$$

where $\kappa$ denotes the epicyclic frequency of the orbits of the stars, $\sigma_{\mathrm{d}}$ is the radial velocity dispersion of the stars, and $\Sigma_{\mathrm{d}}$ the surface density of the disk. $G$ denotes the constant of gravitation. If all other parameters are kept constant, but $\Sigma_{\mathrm{d}}$ is lowered, $Q_{\mathrm{T}}$ rises and the disk becomes more stable against Jeans instabilities. The physical reasoning is that the self-gravity of the disk, which has a destabilizing effect, is reduced by the surrounding halo. Similarly the onset of non-axisymmetric coherent large-scale instabilities of the entire disk such as the bar instability was thought to be damped by a surrounding halo. Ostriker \& Peebles (1973) showed in their classical numerical simulations of the dynamical evolution of a self-gravitating disk that the bar instability could be suppressed, if the disk was embedded in a halo potential. However, modern high-resolution simulations in which the surrounding halo is treated as a dynamically responsive system, have shown that actually the opposite is true. Athanassoula (2002) showed that in her simulations of the bar instability the bar grows stronger, if the disk is embedded in a live dark halo rather than in a static halo potential. This was explained there and, particularly, in Athanassoula (2003) as due to the effect of the live halo on the angular momentum exchange within the galaxy. First doubts about an entirely passive role of the halo were already raised by Toomre (1977). These findings were supported by theoretical studies of the swing amplification of shearing spiral density waves which is also enhanced, if the disk is embedded in a live dark halo instead of a static potential (Fuchs 2004, Fuchs \& Athanassoula 2005). 
Here we return to the severe local Jeans instability of a self-gravitating disk and investigate the effect of the presence of a live dark halo. In the next section we demonstrate how Toomre's concept of the $Q_{\mathrm{T}}$ parameter can be generalized in order to take into account the effect of such a halo. In the final section we discuss implications for realistic disk-halo systems (cf. also Esquivel \& Fuchs 2007).

\section{Modification of the $Q_{\mathrm{T}}$ stability index}

We study the Jeans instability of an infinitesimally thin galactic disk using the model of a patch of the galactic disk developed by Toomre (1964), Goldreich \& Lynden-Bell (1965) and Julian \& Toomre (1966) (cf. also Fuchs 2001). The patch is assumed to rotate around the galactic center and the differential rotation of the stars is approximated as a linear shear flow. The surface density is assumed to be constant over the patch. Polar coordinates are approximated by pseudo Cartesian coordinates $(x, y)$ with $x$ pointing in the radial direction and $y$ in the direction of rotation, respectively. Toomre (1964) has calculated the dynamical response of the disk to a small 'ring-like' perturbation of the gravitational disk potential of the form

$$
\Phi_{\mathrm{k}} \exp i(\omega t+k x)
$$

by solving the linearized Boltzmann equation. The induced density perturbation can be written in the limit $\omega \rightarrow 0$ as

$$
\Sigma_{\mathrm{k}} e^{i k x}=-\frac{\Sigma_{\mathrm{d}}}{\sigma_{\mathrm{d}}^{2}}\left[1-\exp \left(-\frac{k^{2} \sigma_{\mathrm{d}}^{2}}{\kappa^{2}}\right) \cdot \mathrm{I}_{0}\left(\frac{k^{2} \sigma_{\mathrm{d}}^{2}}{\kappa^{2}}\right)\right] \Phi_{\mathrm{k}} e^{i k x},
$$

where $\mathrm{I}_{0}$ denotes the modified Bessel function and $\Sigma_{\mathrm{d}}$ the background surface density of the disk, respectively. In deriving eq. (2.2) a Gaussian velocity distribution of the stars with a velocity dispersion $\sigma_{\mathrm{d}}$ has been adopted. The disk is assumed to be self-gravitating, so that the density - potential pair has to fulfill the Poisson equation implying

$$
\Phi_{k}=-\frac{2 \pi G}{|k|} \Sigma_{k}
$$

Equations (2.2) and (2.3) define together a line in a space spanned by $Q_{\mathrm{T}}$ and the wavelength of the perturbation $\lambda=2 \pi / k$ expressed in units of $\lambda_{\text {crit }}=4 \pi^{2} G \Sigma_{d} / \kappa^{2}$, which separates neutrally stable $\left(\omega^{2} \geqslant 0\right)$ from exponentially unstable $\left(\omega^{2}<0\right)$ perturbations of the disk. The criterion that ensures that all perturbations are neutrally stable is the famous Toomre criterion

$$
Q_{\mathrm{T}} \geqslant 1 \text {. }
$$

The model of a local patch of a galactic disk has been extended by Fuchs (2004) by embedding it into a dark halo. All density gradients in the halo are neglected as in the disk so that the halo density distribution is assumed to be homogeneous. The dark matter particles follow straight-line orbits with an isotropic velocity distribution modelled also by a Gaussian distribution. We can directly apply the results of Fuchs (2004). The dark matter halo responds to the potential perturbation $\Phi_{\mathrm{d}}$ in the disk and develops potential perturbations $\Phi_{\mathrm{h}}$ which have the same radial structure $\exp (i k x)$ as in the disk. From eqns. (26) and (28) of Fuchs (2004) follows that the Fourier coefficients of the potential perturbation in the halo at the midplane of the disk are given by

$$
\Phi_{\mathrm{hk}}=\frac{2 \pi G \rho_{\mathrm{h}}}{\sigma_{\mathrm{h}}^{2}} \frac{1}{k^{2}} \Phi_{\mathrm{dk}}
$$




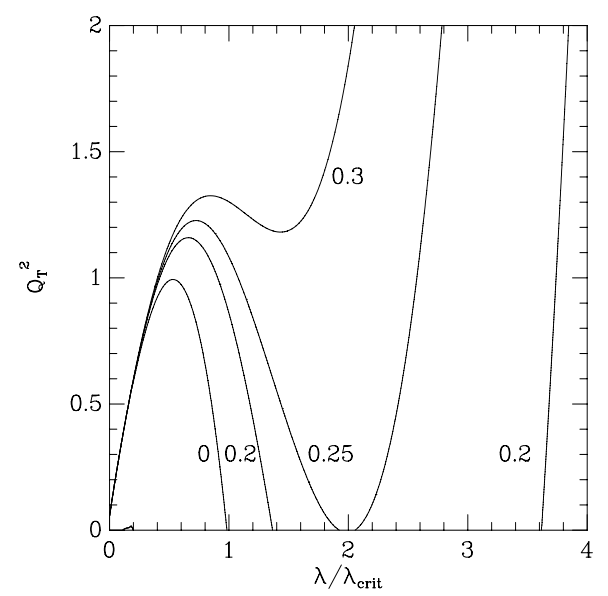

Figure 1. Separation of stable from unstable perturbations of a self-gravitating disk embedded in a live dark halo. $Q_{\mathrm{T}}$ denotes the usual Toomre stability index and $\lambda$ is the wavelength of the perturbation measured in units of $\lambda_{\text {crit }}$. Unstable perturbations are located in the parameter space below the dividing lines. Lines are shown for values of the $\beta$-parameter, which describes the dynamical responsiveness of the dark halo, $\beta=0,0.2,0.25$, and 0.3 , respectively.

where $\rho_{\mathrm{h}}$ and $\sigma_{\mathrm{h}}$ denote the density of the dark halo and the velocity dispersion of the dark matter particles, respectively.

This induced perturbation of the gravitational potential of the dark halo has to be taken into account on the rhs of eq. (2.2),

$$
\Phi_{\mathrm{k}} \rightarrow \Phi_{\mathrm{dk}}+\Phi_{\mathrm{hk}} \propto \Phi_{\mathrm{dk}}
$$

which means that the halo supports the perturbation of the disk and the density perturbation in the disk is stronger than in an isolated disk. Combining eqns. (2.2) to (2.6) leads to an implicit equation that describes the line of neutrally stable $(\omega=0)$ perturbations in the space spanned by $Q_{\mathrm{T}}$ and $\lambda / \lambda_{\text {crit }}$ as in the case of an isolated disk, but now modified by the extra term given in eqns. (2.5) and (2.6). This can be cast into dimensionless form as

$$
\begin{aligned}
\alpha Q_{\mathrm{T}}^{2}=\left[1-\exp \left(-\frac{\alpha Q_{\mathrm{T}}^{2}}{\left(\lambda / \lambda_{\text {crit }}\right)^{2}}\right)\right. & \left.\cdot \mathrm{I}_{0}\left(\frac{\alpha Q_{\mathrm{T}}^{2}}{\left(\lambda / \lambda_{\text {crit }}\right)^{2}}\right)\right] \frac{\lambda}{\lambda_{\text {crit }}} \\
& \times\left(1+\beta\left(\frac{\lambda}{\lambda_{\text {crit }}}\right)^{2}\right)
\end{aligned}
$$

with the parameters $\alpha=(3.36 / 2 \pi)^{2}=0.286$ and $\beta=\left(2 \pi G \rho_{h} / \sigma_{h}^{2}\right) \cdot\left(2 \pi G \Sigma_{d}\right)^{2} / \kappa^{4}$.

In Fig. 1 we illustrate solutions of eq. (2.7) for various values of $\beta$. The case $\beta=0$ reproduces Toomre's classical (1964) result. The unstable perturbations $\left(\omega^{2}<0\right)$ are located in the parameter space below the line. Thus for $Q_{\mathrm{T}} \geqslant 1$ all perturbations are neutrally stable $\left(\omega^{2} \geqslant 0\right)$. This is no longer the case, if finite values of $\beta$ are considered. The graphs of the solutions shown in Fig. 1 always turn upwards at large wavelengths $\lambda$. Thus at large enough wavelengths all perturbations of the disk - halo system become unstable and grow exponentially. This behaviour is related to the Jeans collapse of the halo component. Its Jeans length is given by $\lambda_{\mathrm{J}}=\sqrt{\pi \sigma_{\mathrm{h}}^{2} / G \rho_{\mathrm{h}}}$ or

$$
\frac{\lambda_{\mathrm{J}}}{\lambda_{\text {crit }}}=\frac{1}{\sqrt{2 \beta}} .
$$

In real haloes the Jeans length will be of the order of the size of the halo or even larger, 
Table 1. Local parameters of the Milky Way

\begin{tabular}{ccc}
\hline$\Sigma_{\mathrm{d}}$ & $38 M_{\odot} / p c^{2}$ & (Holmberg \& Flynn 2004) \\
$\sigma_{\mathrm{d}}$ & $40 \mathrm{~km} / \mathrm{s}$ & (Jahreiß \& Wielen 1997) \\
$\kappa$ & $\sqrt{2} \cdot 220 \mathrm{~km} / \mathrm{s} / 8.5 \mathrm{kpc}$ & (flat rotation curve) \\
$\rho_{\mathrm{h}}$ & $0.01 M_{\odot} / p c^{3}$ & (Bahcall \& Soneira 1980) \\
$\sigma_{\mathrm{h}}$ & $220 \mathrm{~km} / \mathrm{s} / \sqrt{2}$ & (isothermal sphere) \\
$\lambda_{\text {crit }}$ & $4.8 \mathrm{kpc}$ & $(\beta=0.0078)$ \\
$\lambda_{\mathrm{J}}$ & $39 \mathrm{kpc}$ & $(\beta)$ \\
\hline
\end{tabular}

because otherwise the haloes would have collapsed to smaller sizes. Thus the inferred instability of the disk - halo system on large scales seems not to occur in real galaxies. As can be seen from Fig. 1 stability on small scales can be ensured by $Q_{\mathrm{T}}$ indices at thresholds which are slightly larger than in isolated disks.

\section{Discussion and Conclusions}

As a first application of the stability criterion derived here we test the stability of the Milky Way disk and the surrounding dark halo in the vicinity of the Sun. The local disk and halo parameters listed in Table 1 imply $Q_{\mathrm{T}}=2.8$ and $\beta=0.0057$, respectively. If we include in our estimate the cold interstellar gas with a local surface density of $4 M_{\odot} / \mathrm{pc}^{2}$ (Dame 1993) and assume a velocity dispersion of the interstellar gas of $\sigma_{\mathrm{g}}=5 \mathrm{~km} / \mathrm{s}$, which leads to a reduced mass weighted effective velocity dispersion of the combined stellar and gaseous disks, the parameter values change to $Q_{\mathrm{T}}=2.2$ and $\beta=0.0078$, respectively. Equation (2.8) implies that $\lambda_{\mathrm{J}}=8 \lambda_{\text {crit }}=39 \mathrm{kpc}$. Thus the Milky Way disk and halo system seems to be very stable.

In order to explore in what range the $\beta$-parameter of spiral galaxies is to be expected, we consider the model of a Mestel disk with the surface density $\Sigma_{d}=\Sigma_{0} R^{-1}$ embedded in a singular isothermal sphere representing the dark halo with the density distribution $\rho_{h}=\rho_{0} R^{-2}$. The rotation curve of the model galaxy is given by

$$
v_{\mathrm{c}}^{2}(R)=v_{\mathrm{d}}^{2}(R)+v_{\mathrm{h}}^{2}(R)
$$

with the disk contribution $v_{\mathrm{d}}^{2}(R)=2 \pi G \Sigma_{\mathrm{d}} R=$ const. and the halo contribution $v_{\mathrm{h}}^{2}(R)=$ $4 \pi G \rho_{\mathrm{h}} R^{2}=$ const. (Binney \& Tremaine 1987). From the radial Jeans equation,

$$
\frac{1}{\rho_{\mathrm{h}}} \frac{d \rho_{\mathrm{h}} \sigma_{\mathrm{h}}^{2}}{d R}=-\frac{v_{c}^{2}}{R},
$$

follows immediately that the velocity dispersion of the dark matter particles is given by

$$
\sigma_{\mathrm{h}}^{2}=\frac{1}{2}\left(v_{\mathrm{d}}^{2}+v_{\mathrm{h}}^{2}\right)
$$

because the particles are bound by both the gravitational disk and halo potentials. We find then

$$
\beta=\frac{v_{\mathrm{h}}^{2}}{R^{2}} \frac{1}{v_{\mathrm{d}}^{2}+v_{\mathrm{h}}^{2}} \frac{R^{2}}{4} \frac{v_{\mathrm{d}}^{4}}{\left(v_{\mathrm{d}}^{2}+v_{\mathrm{h}}^{2}\right)^{2}}=\frac{1}{4} \frac{v_{\mathrm{h}}^{2} v_{\mathrm{d}}^{4}}{\left(v_{\mathrm{d}}^{2}+v_{\mathrm{h}}^{2}\right)^{3}},
$$

which implies the maximal value

$$
\beta \leqslant \beta_{\max }\left(v_{\mathrm{d}}^{2}=2 v_{\mathrm{h}}^{2}\right)=0.037 .
$$

This means that in realistic halo models its density cannot be increased, on one hand, and the velocity dispersion of the halo particles lowered, on the other hand, indiscriminately, 


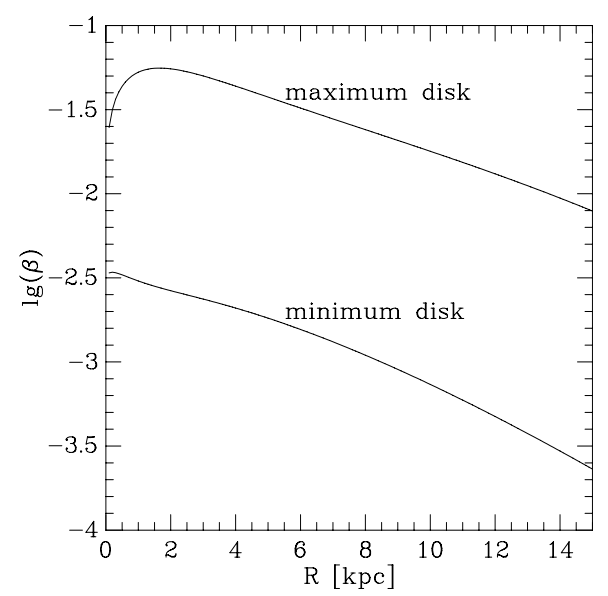

Figure 2. $\beta$-parameters of mass models of the low surface brightness galaxy F568-1.

because the halo has to stay in radial hydrostatic equilibrium. Equation (3.5) implies $\lambda_{\mathrm{J}}=3.7 \lambda_{\text {crit }}$. In order to ensure stability at smaller wave lengths the Toomre stability index must be larger than $Q_{\mathrm{T}} \geqslant 1.02$.

As another concrete example we analyse the dynamics of the low surface brightness galaxy F568-1. De Blok et al. (2001) have observed its rotation curve and constructed mass models for the galaxy. Using the parameters of the models with isothermal haloes and with either a 'realistic' $M / L$-ratio of the disc or a 'maximum-disc', we have solved numerically the radial Jeans equation for the dark matter particles. As shown in Fig. 2 the resulting $\beta$-parameters of both models are consistent with the above estimate.

We conclude from this discussion that embedded galactic disks are not prone to Jeans instabilities, provided their Toomre stability index is a few percent higher than $Q_{\mathrm{T}}=1$. From a practical point of view the destabilizing effect of the surrounding dark halo on the Jeans instability of the embedded galactic disks seems to be negligible.

\section{References}

Athanassoula, E. 2002 ApJ 569, L83

Athanassoula, E. 2003 MNRAS 341, 1179

Bahcall, J. N., \& Soneira, R. M. 1980 ApJ 44, 73

Binney, J., \& Tremaine, S. 1987, Galactic Dynamics (Princeton: Princeton University Press)

Dame, T. M. 1993, in: S. S. Holt and F. Verter (eds.), Back to the Galaxy, AIP Conf. Proc. Vol. 27, (Am. Inst. Phys., New York) p. 267

de Blok, W. J. G., McGaugh, S. S., \& Rubin, V. C. 2001, AJ 122, 2396

Esquivel, O., \& Fuchs, B. 2007 A $\& A$ 468, 803

Fuchs, B. 2001 A $\mathscr{G} A 368,107$

Fuchs, B. 2004 A\&A 419, 941

Fuchs, B., \& Athanassoula, E. 2005, A\&A 444, 455

Goldreich, P., \& Lynden-Bell, D. 1965, MNRAS 130, 125

Holmberg, J., \& Flynn, C. 2004 MNRAS 352, 440

Jahreiß, H., \& Wielen, R. 1997, in: B. Battrick, M.A.C. Perryman \& P.L. Bernacca ( eds.), HIPPARCOS '97' (ESA SP-402, Noordwijk) p. 675

Julian, W. H., \& Toomre, A. 1966 ApJ 146, 810

Ostriker, P. J., \& Peebles, J. P. E. 1973 ApJ 186, 467

Toomre, A. 1964 ApJ 139, 1217

Toomre, A. 1977 ARAA 15, 437 